\title{
Modo de apropiación de la naturaleza en las unidades de producción campesinas de amaranto y maíz en Tochimilco, Puebla, México
} Nature appropriation mode in amaranth and corn production peasant units in Tochimilco, Puebla, Mexico

Vianii Cruz López ${ }^{1}$, Ignacio Ocampo Fletes ${ }^{1}$, José Pedro Juárez Sánchez ${ }^{1}$, Adrián Argumedo Macías $^{1}$, Ernesto Castañeda Hidalgo ${ }^{2}$

Palabras Clave: formas de transformación; intercambios socio-ecológicos; agricultura familiar; cultivos tradicionales

Keywords: transformation ways; socio-ecological exchanges; family farming; traditional crops

Recepción: 09-12-2017 / Aceptación: 08-03-2018

\section{Resumen}

Para satisfacer sus necesidades el hombre tiene que apropiarse de la naturaleza. De los dos modos contrapuestos de apropiación, el campesino y el agroindustrial, el menos degradante es el primero, además de ser el más importante por el volumen de alimentos producidos, el trabajo generado y las ventajas ecológicas. Por la relevancia de este modo de apropiación, se planteó como objetivo mostrar la pertinencia ecológica, social y económica del modo de apropiación campesino y las características que propician tendencia al agroindustrial. El estudio se realizó en las comunidades San Lucas Tulcingo y La Magdalena Yancuitlalpan del municipio de Tochimilco, Puebla, México, seleccionadas por el patrón de paisajes (gradientes de altitud), la superficie sembrada de amaranto y de maíz, y el tipo de tecnología usada. Se analizó la Unidad de Producción Campesina que tiene como base la siembra de amaranto y de maíz. Las técnicas de investigación fueron la encuesta y la observación directa. Se realizó un muestreo estratificado aleatorio, resultando una muestra de 74 productores. Se construyó un Índice de Campesinidad (IC) con base a nueve atributos. Se aplicó una regresión logística por el método de Wald (hacia adelante) para determinar los indicadores con mayor influencia en el Índice de Campesinidad (IC). Los resultados de los nueve atributos muestran que las dos localidades se clasifican en el modo de apropiación campesino. San Lucas Tulcingo presentó mayor empleo de energía fósil por el uso de maquinaria agrícola, mientras que en La Magdalena Yancuitlalpan el modo campesino emplea mayor mano de obra familiar. Tanto la fuente de energía como la fuerza de trabajo manifestaron asociación con el Índice de Campesinidad de acuerdo al procedimiento de selección ${ }^{1}$ Programa en Estrategias para el Desarrollo Agrícola Regional, Colegio de Postgraduados, Campus Puebla. E-mail: agroecología_iof@yahoo.com ${ }^{2}$ Instituto Tecnológico del Valle de Oaxaca, Tecnológico Nacional de México

(C) Universidad De La Salle Bajío (México) 
Modo de apropiación de la naturaleza en las unidades de producción campesinas de amaranto y maíz en Tochimilco, Puebla, México

de variables con el método de Wald (hacia adelante). Se concluye que las Unidades de Producción Campesinas de San Lucas Tulcingo presentaron un Índice de Campesinidad de 0.327 (tendiente al agroindustrial), y las de La Magdalena Yancuitlalpan de 0.298, pero ambas conservan su modo de apropiación campesino. La diferencia está en la principal fuente de energía usada; energía fósil y energía de trabajo familiar, respectivamente.

\begin{abstract}
In order to satisfy his needs, the man has to appropriate the nature. Of the two opposing modes of appropriation, the peasant and the agroindustrial, the least degrading is the first, in addition to being the most important because the volume of food produced, work generated and ecological advantages. Due the relevance of this appropriation mode, the objective was to show the ecological, social and economic relevance of the peasant appropriation mode, and the characteristics that propitiate the agroindustrial trend. The study was carried out in the communities of San Lucas Tulcingo and La Magdalena Yancuitlalpan of Tochimilco municipality in Puebla, Mexico, selected by the landscapes pattern (altitude gradients) the area with amaranth and corn seeded and the type of technology. The Peasant Production Unit (PPU) was analyzed, which is based on the sowing of amaranth and corn. The research techniques were the survey and the direct observation. A stratified random sampling was performed, resulting in a sample of 74 producers. A Peasantry Index (CI) was constructed based on nine attributes. A logistic regression was applied by the Wald method (forward) to determine the indicators with the greatest influence on the Peasantry Index (CI). The results of the nine attributes show that the two localities are classified in the mode of peasant appropriation. San Lucas Tulcingo presented greater use of fossil energy by the use of agricultural machinery, while in La Magdalena Yancuitlalpan the peasant mode employs more family labor. Both, the energy source and the labor force, showed association with the peasantry index according to the variable selection procedure, the forward Wald method. It is concluded that the PPU of San Lucas Tulcingo presented a Peasantry Index of 0.327 (tending to the agroindustrial), and those of La Magdalena Yancuitlalpan of 0.298 , but both conserved their mode of peasant appropriation. The difference is in the main source of energy used; fossil energy and family work energy, respectively.
\end{abstract}




\section{Introducción}

La naturaleza es la proveedora de las materias primas necesarias para la producción y reproducción social (Carrasco 2006, 57), por ello, los seres humanos la afectan al apropiarse de sus elementos y también al devolverlos ya socializados (Toledo et al. 2002a, 22). Este metabolismo lo realizan los seres humanos a través del proceso social del trabajo, por el cual se apropian, producen, circulan, transforman, consumen y excretan productos, materiales, energía y agua, provenientes del mundo natural (Toledo et al. 2002b, 12).

En el sector agropecuario existen dos modos radicalmente diferentes de apropiación de la naturaleza, el campesino ${ }^{1}$ y el agroindustrial ${ }^{2}$ (Toledo et al. 2002a, 34). El modo campesino se caracteriza por practicar una agricultura de corte minifundista, por su relativo grado de autosuficiencia gracias a la diversidad productiva que posee, por estar basado en el trabajo familiar con un mínimo de inputs externos y por buscar la reproducción de la unidad de producción y no su mercantilización (Toledo 1992, 1). Por el contrario, la agricultura industrial se caracteriza por la búsqueda de ganancias mediante la transformación de capital natural en capital económico y por poseer una alta dependencia de insumos y de mano de obra remunerada (Gallopín et al. 1995, 39).

De acuerdo a las características de los modos de apropiación de la naturaleza, se considera que la agricultura campesina tiene menor impacto sobre los agroecosistemas y posee una gran flexibilidad y creatividad para readecuar sus estrategias (Cáceres et al. 2010, 113) lo que explica la compatibilidad del modelo campesino con las comunidades indígenas, en donde existen identidades socioculturales en las que el medio ambiente y la biosfera ha permanecido por encima del sistema social, y éste por encima del sistema económico (Vara y Cuéllar 2013, 6).

Los campesinos y comunidades indígenas han construido un espacio social, en ellas, destacan las formas de manejo de los recursos naturales, tienen cierta autonomía de los mercados, establecen criterios de cohesión social y solidaridad, tienen una racionalidad ecológica que

\footnotetext{
${ }^{1}$ Clase social estudiada exhaustivamente por Wolf (1982), Shanin (1983), Chayanov (1985), entre otros.

${ }^{2}$ El modo agroindustrial también llamado moderno tiene sus orígenes en Occidente y de la revolución industrial y científica que tuvo lugar en Europa y otros países templados a partir del siglo dieciocho. Este modo de apropiación de la naturaleza surge desde el mundo urbano-industrial para generar los alimentos, materias primas y energías requeridas en los espacios no rurales de la tierra (Toledo et al., 2002a, 34-35). Fue una transformación de lo campesino a lo agroindustrial a partir del uso de nuevas fuentes de energía (carbón, petróleo y gas) provocando la extracción de bienes de la naturaleza, el incremento en la productividad del trabajo, el aumento de la escala de producción, la especialización de los productores que garantizó la producción para las ciudades, aumentó su dependencia de insumos externos y la consolidación de la agroindustria (Toledo et al. 2002a, 35-36, citando a Debeir et al. 1986 y Smil, 1994).
} 
respeta los límites de la naturaleza, y el trabajo humano se orienta a garantizar y mantener la capacidad productiva del agroecosistema de la que depende su modo de vida (Sevilla y Soler 2009, 38).

Por el contrario, la agricultura industrial busca acelerar el proceso de producción agrícola para generar los alimentos, materias primas y energías requeridas que demandan los espacios urbano-industrial (Martínez 2002, 39) con el fin de obtener mayores rendimientos, pero genera agroecosistemas inestables que apuntan a la simplificación, fragilidad y homogeneidad de los paisajes agrícolas, así como al sometimiento a tensiones ambientales locales y vicisitudes económicas difíciles de comprender y controlar (Rappaport 1975, 389; Dal Soglio 2016, 15). Otros efectos de la agricultura industrial son la pérdida de biodiversidad, uso múltiple de los recursos y de conocimientos específicos propios a cada cultivo, clima, suelo, contexto social y cultural (Camejo 2016, 58; Casado et al. 2000, 41), además de la degradación y polución progresiva de los recursos naturales (Cáceres et al. 2010, 114). Se puede decir que la sostenibilidad de la agricultura campesina depende de las relaciones sociales y los procesos ecológicos; y estas relaciones y procesos son diferentes de los asociados con la producción agropecuaria mercantilista (Sevilla y Woodgate 2013, 30).

El proceso de modernización de la agricultura industrial que inició a mediados del siglo pasado, significó para los habitantes del medio rural que practican la agricultura campesina, el sometimiento a un proceso de transformación en la forma de manejar los recursos naturales (Acosta 2008, 1). La transformación ha sido posible gracias a tecnologías que impulsan los monocultivos, como la mecanización, el mejoramiento genético de variedades, el desarrollo de agroquímicos y la transferencia de conocimientos generados en centros de investigación (Altieri y Nicholls 2000, 114; Sarandón 2011, 5). Eso indica que el modelo de producción de monocultivos privilegia el uso eficiente de los insumos externos y la mecanización, la flora y la fauna silvestres son competidores directos de los productos cosechados, y por lo tanto son eliminados (Suso et al. 2013, 10).

Por ello, se considera que la agricultura convencional, en parte es responsable de los impactos en los ecosistemas, ya que su intensificación ha sido el resultado de la disponibilidad de energías fósiles relativamente de bajo costo, que han permitido la mecanización y la producción de fertilizantes inorgánicos y otros insumos a gran escala, así como la utilización de variedades de cultivos adaptadas a estos insumos (Hernández 2013,1). Precisamente, los sistemas agrícolas 
han transitado de complejos, al ser formados por un mosaico de cultivos y con una proporción elevada de hábitats naturales y seminaturales, a paisajes dominados por extensas áreas cultivadas con escaza heterogeneidad espacial (Gabriel et al. 2006, 2011).

A pesar de la modernización agrícola, la importancia de la agricultura campesina se mantiene gracias a su contribución en la producción de alimentos y a la conservación de los ecosistemas y agroecosistemas. De acuerdo con la Organización de las Naciones Unidas para la Alimentación y la Agricultura (FAO 2015, 33) a escala mundial más del 90\% de las unidades de producción agrícolas son campesinas y producen más del $80 \%$ de alimentos en $12 \%$ de la superficie agrícola. En México las cifras son similares, $81.3 \%$ unidades campesinas en $8.8 \%$ de la superficie total de labor generan 3.5 millones de empleos familiares, 5.1 millones de jornaleros y $39 \%$ de los alimentos que consume el país (SAGARPA y FAO 2012, 52, 58). Estos grupos campesinos son altamente conservadores de los recursos naturales, ya que existe una clara correspondencia entre las áreas de bosque tropical y la presencia de pueblos indígenas que practican la agricultura campesina en América Latina, la cuenca del Congo en África y varios países de Asia (Toledo y Barrera 2008, 53).

Los atributos o rasgos que diferencian los modos de apropiación campesino y agroindustrial son: 1) el tipo de energía usada en el proceso de producción, 2) la escala de las actividades productivas, 3) el grado de autosuficiencia de la unidad productiva, 4) su nivel de fuerza de trabajo, 5) el grado de diversidad (eco-geográfica, productiva, biológica, genética), 6) su nivel de productividad ecológica o energética, 7) su nivel de productividad del trabajo, 8) el tipo de conocimientos empleados durante la apropiación/producción, y 9) la visión del mundo (natural y social) de la racionalidad productiva (Toledo et al. 2002a, 37-38).

Debido a la importancia de apropiación del modo campesino, del volumen de alimentos producidos y empleos generados, de sus ventajas ecológicas, frente al modo de apropiación agroindustrial que con el fin de comprender y mantener vivo el modo de apropiación campesino bajo un marco de soberanía alimentaria, entendiendo ésta como el derecho de los pueblos a decidir su política agrícola y alimentaria para tener acceso a una alimentación inocua, nutritiva y culturalmente apropiada, y a los recursos necesarios para la producción de dichos alimentos (Ortega y Rivera 2010, 55), donde los seres humanos tienen el control sobre cómo comer y alimentarse, cómo usar la tierra, el agua y otros recursos para el beneficio de las generaciones actuales y futuras (European Coordination Vía Campesina 2018, 3), se considera relevante 
identificar los elementos que dan fortaleza al modo de apropiación campesino y los elementos que contribuyen a su desplazo hacia el modo de apropiación agroindustrial. Sin embargo, este proceso ni es total ni es completo, ya que existen fuerzas de resistencia campesina frente a las fuerzas modernizadoras (Toledo 1999, 12). Son estrategias "defensivas" basadas en la redefinición del diseño productivo de sus explotaciones, como parte de los procesos de acomodación activa de los campesinos a una realidad en permanentemente transformación, que a través de sus capacidades de flexibilidad y creatividad readecuan sus estrategias (Cáceres et al. 2010, 109, 112-113).

Por lo anterior, el objetivo del estudio fue mostrar la pertinencia ecológica, social y económica del modo de apropiación campesino y las características que propician tendencia al agroindustrial, en unidades de producción de amaranto y de maíz en las comunidades La Magdalena Yancuitlalpan y San Lucas Tulcingo del municipio de Tochimilco, Puebla, México.

\section{Metodología}

El estudio se realizó en el municipio de Tochimilco, Puebla, que se encuentra a una altitud entre 1 800 y $5500 \mathrm{msnm}$ (cumbre del Popocatépetl), el clima predominante es $\mathrm{C}(\mathrm{w} 1)$ (templado subhúmedo con lluvias en verano), su temperatura oscila entre los 12 y $18^{\circ} \mathrm{C}$. Colinda al norte con el municipio de San Nicolás de los Ranchos, al sur con los municipios de Atzitzihuacán y Cohuecán; al este con los municipios de Tianguismanalco y Atlixco y al oeste con el estado de Morelos y el volcán Popocatépetl. Respecto al uso del suelo, 44\% es agrícola y ganadero, $43 \%$ bosque y en porcentajes similares al 3\% zona urbana, área sin vegetación aparente, pastizal y selva. Predominan los suelos regosoles, phaeozem y andosoles (INAFED 2010; INEGI 2010, 1). Para el 2010 la población fue de 17028 personas y 3885 viviendas habitadas en promedio por 4.4 personas (CONEVAL 2010, 1).

El municipio tiene una actividad preponderantemente agrícola. De acuerdo con el Sistema de Información Agroalimentaria y Pesquera (SIAP 2014) la superficie sembrada en el ciclo 2014 fue de 5941 hectáreas, de las cuales $82.8 \%$ son de temporal. El principal cultivo sembrado fue maíz para grano (2 820 ha); en segundo lugar, el cultivo de amaranto (1 097 ha). Otros cultivos de importancia son: frijol, chía, tomate verde y calabaza; frutales como aguacate, tejocote y pera, y flores entre las que destaca alhelí, nube y gladiola. 
Para este estudio se seleccionaron dos localidades del municipio: La Magdalena Yancuitlalpan ubicada a $2400 \mathrm{msnm}$, entre las coordenadas $18^{\circ} 54^{\prime} 35^{\prime \prime}$ latitud norte y $98^{\circ} 35^{\prime} 07^{\prime \prime}$ longitud oeste y San Lucas Tulcingo localizada a 1900 msnm, entre las coordenadas 1850`21”' latitud norte y 98 $35^{\prime} 43^{\prime \prime}$ longitud oeste (Mapa 1). Se eligieron con base en los criterios de superficie sembrada de amaranto y de maíz, los patrones del paisaje en relación a su gradiente de altitud, y considerando la sugerencia de Gliessman (2002, 290) relacionado con prácticas agroindustriales o tradicionales. Esta última supone que espacios con poca pendiente están más expuestos a la modernización agrícola, sobre todo, a la homogenización del agroecosistema (monocultivo) y a la tecnificación (introducción de maquinaria), en relación a espacios con pendientes más pronunciadas. En el caso estudiado, San Lucas Tulcingo se ubica en la primera condición, mientras que La Magdalena Yancuitlalpan se localiza en condiciones de pendientes más abruptas en las laderas del Volcán Popocatépetl.

A través de la investigación no experimental-transversal de tipo explicativa (Hernández et al. 2006, 99, 155) se establecieron las características que causan la continuación del modo de apropiación campesino y las que propician tendencia al modo de apropiación agroindustrial. El objeto de estudio fue la Unidad de Producción Campesina (UPC) donde la familia siembra entre sus principales cultivos el amaranto y el maíz. De acuerdo a Shanin $(1983,54)$ "la unidad doméstica campesina se caracteriza por una casi total integración de la vida de la familia y su explotación agrícola. La familia suministra el trabajo necesario mientras que las actividades agrícolas se orientan, principalmente, a la producción suficiente para satisfacer sus necesidades básicas y los tributos impuestos por los poseedores del poder económico y político."

Al respecto Chayanov $(1985,47)$ señala que uno de los factores principales de la organización de la unidad económica campesina es el carácter de la familia, que no contrata fuerza de trabajo, la composición y el tamaño de la familia determinan el monto de la fuerza de trabajo, su composición y el grado de actividad. Wolf $(1982,24)$ considera que la unidad campesina no es solo una organización productora formada por una cantidad de manos que realizan el trabajo del campo, también forma una unidad de consumo con igual o más bocas que trabajadores a los que se debe facilitar otros servicios. 
Modo de apropiación de la naturaleza en las unidades de producción campesinas de amaranto y maíz en Tochimilco, Puebla, México

Mapa 1. Localización de San Lucas Tulcingo y La Magdalena Yancuitlalpan en el municipio de Tochimilco, Puebla, México.

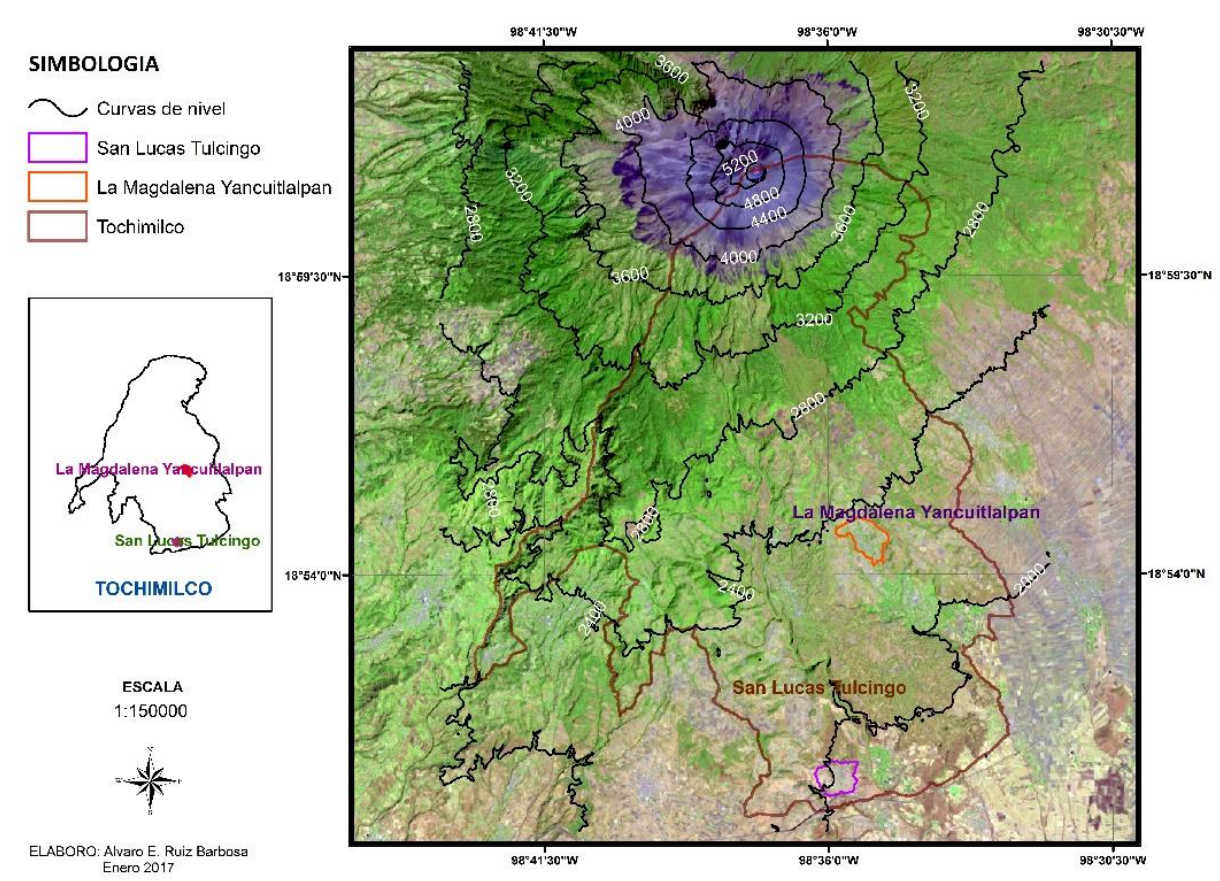

Fuente: Elaboró Ruiz Barbosa Á. E. (2017) a partir de:

INEGI (2017). Marco geoestadístico-Datos vectoriales topográficos, escala 1:150,000.

Las técnicas empleadas para obtener la información de campo fueron la encuesta y la observación directa. Como instrumentos se emplearon el cuestionario estructurado y una guía de observación para cada técnica respectivamente (Rojas 2013, 202). La obtención de la información se realizó en mayo y junio de 2016 para generar datos del ciclo agrícola 2015.

Se consideró como marco de muestreo la población de productores que siembran amaranto (como uno de los principales cultivos en sus Unidades de Producción), resultando 150 en La Magdalena Yancuitlalpan y 184 en San Lucas Tulcingo. El censo de productores que sembró amaranto en cada comunidad se obtuvo a través de registros con informantes clave para la primera localidad y con las autoridades ejidales para la segunda. El tamaño de la muestra se determinó utilizando el muestreo estratificado aleatorio con distribución de Neyman cuya ecuación se presenta a continuación:

$$
n=\frac{\left(\sum_{t=1}^{k} N_{i} s_{i}\right)^{2}}{N^{2} V+\sum_{i=1}^{k} N_{i} s_{i}^{2}}
$$


Donde:

$\mathrm{n}=$ tamaño de muestra.

$\mathrm{N}=$ Tamaño de la población (334 productores).

$\mathrm{Ni}=$ Tamaño del estrato $\mathrm{i}$ (estrato $1=150$; estrato $2=184$ productores).

$\mathrm{Si}^{2}=$ Varianza (superficie) del estrato i (estrato $1=2.2 \mathrm{ha}$; estrato $2=2.22 \mathrm{ha}$ ).

$\mathrm{d}=$ Precisión (fijada en 0.25 ha).

$\mathrm{Z} \alpha / 2=$ Confiabilidad (valor de $\mathrm{z}$ de tablas con $\alpha=0.95 ; \mathrm{z}=1.96$ ).

$\mathrm{k}=$ Número de estratos (2).

La ecuación empleada para distribuir el tamaño de la muestra entre los estratos fue la siguiente:

$$
n_{i}=\frac{N_{i} s_{i}}{\sum_{i=1}^{k} N_{i} s_{i}} n
$$

La varianza de cada localidad se obtuvo considerando las superficies reportadas por cada productor en el listado de beneficiarios de 2016, del Programa de Apoyos Directos al Campo (PROCAMPO) implementado por la SAGARPA (2016).

El tamaño de la muestra fue:

$\mathrm{n}=74$ productores.

$\mathrm{ni}_{1}=33$ productores en La Magdalena Yancuitlalpan.

$\mathrm{ni}_{2}=41$ productores en San Lucas Tulcingo.

El Índice de Campesinidad (IC) se basó en la variable modo de apropiación de la naturaleza, a partir de la metodología propuesta por Toledo et al. (2002a, 62, 111) que propone la aplicación de cuestionarios en los estudios a nivel localidad para recabar información de los indicadores que se muestran en el Tabla 1. Para la construcción del Índice de Campesinidad, se consideró el promedio de cada uno de los indicadores a los que se les asignó un valor entre 0 y 1 , donde 0 representa al modo de apropiación campesino y 1 al modo de apropiación agroindustrial. 
Modo de apropiación de la naturaleza en las unidades de producción campesinas de amaranto y maíz en Tochimilco,

Puebla, México

Tabla 1. Indicadores y subindicadores seleccionados por atributo para evaluar el modo de apropiación de la naturaleza en Unidades de Producción Campesina del municipio de

Tochimilco, Puebla, México.

\begin{tabular}{|c|c|c|c|c|}
\hline Atributo & $\begin{array}{c}\text { Indicador y } \\
\text { subindicadores }\end{array}$ & Operacionalización & \multicolumn{2}{|l|}{ Codificación } \\
\hline \multirow[t]{8}{*}{ Energía } & a) Doméstica & \multirow{3}{*}{ Fuente de energía para cocinar } & Gas & 1 \\
\hline & & & Gas y leña & 0.5 \\
\hline & & & Leña & 0 \\
\hline & b) Productiva & \multirow{3}{*}{$\begin{array}{l}\text { Fuente de energía productiva por } \\
\text { labor }\end{array}$} & Mecánica & 1 \\
\hline & & & \multirow{4}{*}{$\begin{array}{l}\text { Mecánica-humana } \\
\text { Animal } \\
\text { Animal-Humana } \\
\text { Humana }\end{array}$} & 0.75 \\
\hline & & & & 0.5 \\
\hline & & \multirow[t]{2}{*}{ Número de labores } & & \multirow{2}{*}{$\begin{array}{l}0.25 \\
0\end{array}$} \\
\hline & & & & \\
\hline Escala & Tamaño de la UP & Superficie/10 & Valor resultante & - \\
\hline \multirow[t]{7}{*}{ Dependencia } & a) Alimentaria & $\begin{array}{c}\left.\text { (Integrantes de la familia } * 215 \mathrm{~kg}^{1}\right) \text { - } \\
(\% \text { cubierto con la producción de la } \\
\text { UP) }\end{array}$ & \multirow[t]{2}{*}{$\begin{array}{l}\text { Valor resultante } \\
\text { de la fórmula }\end{array}$} & \multirow[t]{2}{*}{ - } \\
\hline & \multicolumn{2}{|r|}{100} & & \\
\hline & b) Productiva & \multirow{5}{*}{$\begin{array}{c}\text { Promedio de insumos } \\
\text { externos utilizados durante la } \\
\text { producción de amaranto y de maíz }\end{array}$} & 4 insumos & 1 \\
\hline & & & 3 insumo & 0.75 \\
\hline & & & 2 insumos & 0.5 \\
\hline & & & 1 insumo & 0.25 \\
\hline & & & 0 insumos & 0 \\
\hline \multirow{9}{*}{$\begin{array}{l}\text { Fuerza de } \\
\text { Trabajo }\end{array}$} & \multirow{9}{*}{$\begin{array}{l}\text { Tipo de fuerza de } \\
\text { trabajo }\end{array}$} & \multirow{5}{*}{$\begin{array}{l}\text { Mano de obra no remunerada y } \\
\text { Remunerada en amaranto }\end{array}$} & Remunerada & 1 \\
\hline & & & +Remunerada & 0.75 \\
\hline & & & $\begin{array}{l}\text { Remunerada }=\mathrm{No} \\
\text { remunerada }\end{array}$ & 0.5 \\
\hline & & & +No remunerada & 0.25 \\
\hline & & & No remunerada & 0 \\
\hline & & \multirow{4}{*}{$\begin{array}{l}\text { Número de labores en las que se } \\
\text { contrata mano de obra }\end{array}$} & 4 labores & 1 \\
\hline & & & 3 labores & 0.75 \\
\hline & & & 2 labores & 0.5 \\
\hline & & & 1 labor & 0.25 \\
\hline \multirow[t]{6}{*}{ Diversidad } & \multirow[t]{5}{*}{ a) Agrícola } & $\begin{array}{l}\text { Diversidad de especies (Número de } \\
\text { cultivos) }\end{array}$ & \multicolumn{2}{|l|}{$\begin{array}{c}\text { (No. de cultivos } * \\
\left.\text { Valor por especie }{ }^{2}\right)- \\
1\end{array}$} \\
\hline & & \multirow{4}{*}{$\begin{array}{l}\text { Diversidad dentro de especies de } \\
\text { amaranto y de maíz }\end{array}$} & 1 variedad & 0.75 \\
\hline & & & 2 variedades & 0.5 \\
\hline & & & 3 variedades & 0.25 \\
\hline & & & 4 variedades & 0 \\
\hline & b) Pecuaria & $\begin{array}{l}\text { Especies animales } \\
\text { manejadas en la UP }\end{array}$ & $\begin{array}{l}\text { (No. de especies * } \\
\left.\text { Valor por especie }{ }^{2}\right)- \\
1\end{array}$ & \\
\hline \multirow[t]{2}{*}{$\begin{array}{l}\text { Productividad } \\
\text { del trabajo }\end{array}$} & $\begin{array}{l}\text { Productividad del } \\
\text { trabajo del } \\
\text { amaranto }\end{array}$ & $\begin{array}{l}\text { Rendimiento } \mathrm{tha}^{-1} / \mathrm{J} \text { ornales } \\
\text { empleados en la producción de } \\
\text { amaranto } \\
\end{array}$ & \multirow{2}{*}{$\begin{array}{l}\text { Promedio de los } \\
\text { valores resultantes } \\
\text { de las fórmulas } \\
\text { aplicadas }\end{array}$} & \multirow[b]{2}{*}{-} \\
\hline & $\begin{array}{l}\text { Productividad del } \\
\text { trabajo del maíz }\end{array}$ & $\begin{array}{l}\text { Rendimiento t ha }{ }^{-1} / \text { Jornales } \\
\text { empleados en la producción de maíz }\end{array}$ & & \\
\hline $\begin{array}{l}\text { Productividad } \\
\text { energética }\end{array}$ & $\begin{array}{l}\text { Eficiencia } \\
\text { Energética por }\end{array}$ & $\begin{array}{c}\text { Aporte energético de amaranto/ } \\
\text { Energía empleada para la producción }\end{array}$ & & \\
\hline
\end{tabular}




\begin{tabular}{|c|c|c|c|c|}
\hline & $\begin{array}{l}\text { hectárea de } \\
\text { amaranto }\end{array}$ & de amaranto & \multirow{2}{*}{$\begin{array}{l}\text { Promedio de los } \\
\text { valores resultantes } \\
\text { de las fórmulas } \\
\text { aplicadas }\end{array}$} & \multirow[t]{2}{*}{-} \\
\hline & $\begin{array}{l}\text { Eficiencia } \\
\text { Energética por } \\
\text { hectárea de maíz }\end{array}$ & $\begin{array}{c}\text { Aporte energético de maíz }{ }^{3} \text { / Energía } \\
\text { empleada para la producción de } \\
\text { maíz }^{4}\end{array}$ & & \\
\hline Conocimiento & $\begin{array}{l}\text { Tipo de } \\
\text { conocimiento }\end{array}$ & $\begin{array}{l}\text { Fuente de conocimiento: técnica } \\
\text { (exógena) o empírica (local) }\end{array}$ & $\begin{array}{l}\text { Exógeno aplicado } \\
\text { Exógeno no } \\
\text { aplicado } \\
\text { Endógeno }\end{array}$ & $\begin{array}{l}1 \\
0.5 \\
0\end{array}$ \\
\hline Cosmovisión & $\begin{array}{l}\text { Rituales o } \\
\text { creencias en torno } \\
\text { al cultivo de } \\
\text { amaranto y de } \\
\text { maíz }\end{array}$ & Número de tradiciones 5 & $\begin{array}{l}0 \text { tradiciones } \\
1 \text { tradición } \\
2 \text { tradiciones } \\
3 \text { tradiciones }\end{array}$ & $\begin{array}{l}1 \\
0.4 \\
0.2 \\
0\end{array}$ \\
\hline $\begin{array}{l}{ }^{1} \text { El con } \\
\text { kg. } \\
{ }^{2} \text { Valor } 1 \\
{ }^{3} \text { Aporte } \\
{ }^{4} \text { Energí } \\
\text { acuerdo } \\
2002 a, C \\
{ }^{5} \text { Para ar }\end{array}$ & $\begin{array}{l}\text { Imo per cápita anua } \\
\text { r especie }=1 / \text { moda } \\
\text { nergético por hectár } \\
\text { empleada para la pr } \\
\text { 1 tipo de tracción*en } \\
\text { ). } \\
\text { bos cultivos se consi }\end{array}$ & $\begin{array}{l}\text { alculado con los datos obtenidos en la } \\
\text { especies. } \\
\text { de maíz reportado por Menchú y Mén } \\
\text { ucción de una hectárea de maíz = \% d } \\
\text { gía empleada por hectárea por tipo de } \\
\text { raron las prácticas comunes en las cre }\end{array}$ & $\begin{array}{l}\text { Investigación fue } 215 \\
\text { ez }(2007,48) \text {. } \\
\text { labores realizadas de } \\
\text { cacción (Toledo et al. } \\
\text { cias de las familias. }\end{array}$ & \\
\hline
\end{tabular}

El procedimiento para calcular cada uno de los atributos descrito en la Tabla 1, fue el siguiente, en el que las escalas van de 0 a 1 , donde 0 se considera modo de apropiación campesino y 1 modo de apropiación agroindustrial:

Fuente de energía. Se calculó considerando los indicadores fuente de energía doméstica empleada en las unidades de producción UP, y fuente de energía productiva en las labores realizadas para los cultivos de amaranto y de maíz. En el caso fuente de energía productiva se consideraron dos sub-indicadores más: fuente de energía productiva en amaranto y fuente de energía productiva en maíz. Para cada cultivo se asignó un valor a cada labor de acuerdo a la tecnología empleada. El valor del sub-indicador fuente de energía productiva en amaranto se calculó de la siguiente manera: valor total de la tecnología empleada dividido entre número de labores, dando como resultado 0.575 .

Para el cálculo de la fuente de energía del maíz se siguió el mismo procedimiento. Los valores de ambos cultivos se promediaron dando como resultado fuente de energía productiva $($ FEA + FEM/2). Para el calculó de energía se sumaron los valores promedios de fuente de energía doméstica más fuente de energía productiva (FE = FED + FEP).

Escala. Para definir el valor de la escala se consideró la superficie (ha) que posee la unidad de producción campesina y se dividió entre 10 para obtener valores entre 0 y 1 . 
Dependencia. Se calculó dependencia alimentaria de maíz y dependencia productiva de amaranto y de maíz promediando los valores de cada uno. Para la dependencia alimentaria en maíz (producto de autoconsumo) se calculó el consumo total anual de la unidad de producción de acuerdo al número de integrantes, resultando un consumo per cápita anual de $215 \mathrm{~kg}$; a este valor se le restó la cantidad de maíz producida por la unidad de producción y se dividió entre 100 para estimar el rango entre 0 y 1 . Para el amaranto (producto para el mercado) no se calculó dependencia alimentaria por el bajo consumo en todas las unidades de producción.

Para la dependencia productiva, a cada unidad de producción se les asignaron valores de acuerdo al número de insumos empleados en ambos cultivos y se promediaron. Los insumos considerados fueron: semilla, fertilizante, plaguicida y herbicida.

Fuerza de trabajo. Para el amaranto se calculó el número de jornales remunerados y no remunerados en cada unidad de producción. Para el maíz se consideró el número de labores en las que se contrata mano de obra.

Diversidad. Se consideró la diversidad de especies y diversidad dentro de especies agrícolas (ambos cultivos) y pecuarias. Para la diversidad agrícola (diversidad de especies) se asignó un valor por especie cultivada dividiendo 1 entre el máximo número de especies encontradas; sin embargo, finalmente se optó por tomar la moda en lugar del máximo por su representatividad. Posteriormente este valor se multiplicó por el número de especies, resultando que las unidades de producción con más especies tenían un valor mayor de acuerdo al Índice de Campesinidad. Un valor cercano a uno era agroindustrial y cercano a cero era campesino, por ello, para invertir los valores, a uno se le resto el resultado obtenido. La diversidad pecuaria se calculó de la misma forma que la agrícola considerando especies animales.

Productividad. Considerando que la productividad es la relación entre lo producido y los medios empleados, para la productividad del trabajo se consideró la relación entre el rendimiento obtenido en toneladas por hectárea $\left(\mathrm{t} \mathrm{ha}^{-1}\right)$ y el número de jornales empleados para la producción de esa superficie y el resultado indica las toneladas producidas por cada jornal. Mientras más agroindustrial sea el sistema agrícola se espera que la productividad del trabajo sea mayor, es decir, mayor número de toneladas producidas por un jornal.

Por otra parte, la productividad energética expresa la cantidad de kilocalorías producidas por cada kilocaloría invertida. Para el caso en estudio se multiplicó el rendimiento por hectárea en kilogramos por las kilocalorías contenidas en un kilogramo de amaranto y de maíz, el 
resultado se dividió entre las kilocalorías invertidas para la producción de una hectárea de amaranto y de maíz considerando el porcentaje de labores realizadas con maquinaria, yunta y fuerza humana, así como las kilocalorías que se requieren por hectárea de cada una de estas prácticas. Los datos de las kilocalorías por cada tipo de tecnología se tomaron de la propuesta de Toledo et al. (2002a, 95).

Conocimiento. A cada unidad de producción campesina se le asignó un valor de acuerdo a la asesoría técnica recibida y adopción de las prácticas por dicha asesoría (exógeno aplicado, exógeno no aplicado y endógeno).

Cosmovisión. Se estimó considerando el número de tradiciones o costumbres en torno a la producción agrícola en el amaranto y en el maíz. Para ambos cultivos se consideran las mismas prácticas: $i$ ) siembra y cosecha de acuerdo a las fases de la luna, ii) bendición de semillas el día de la Candelaria (2 de febrero), y iii) floreo de parcelas el día de San Miguel Arcángel (se realiza el 28 de septiembre para que esté preparado para el 29 de septiembre).

Para analizar la información se recurrió a la estadística paramétrica y no paramétrica y se aplicó una regresión logística por el método de Wald (hacia adelante) para determinar los indicadores con mayor influencia en el Índice de Campesinidad (IC).

\section{Resultados}

Características de las Unidades de Producción Campesinas (UPC):

\section{Composición}

Las UPC estudiadas en La Magdalena Yancuitlalpan y en San Lucas Tulcingo muestran similitudes. Están compuestas en promedio por cinco personas, lo cual coincide con lo reportado por Méndez $(2011,75)$. En el caso de la primera comunidad 3.7 integrantes y en la segunda 2.9 se involucran en la actividad de la UPC de forma directa y constante. En La Magdalena Yancuitlalpan, en $46 \%$ de las UPC y en San Lucas Tulcingo en $43.9 \%$ al menos un individuo trabajó fuera de la misma. Esto denota la complementariedad de las actividades. En La Magdalena Yancuitlalpan, $48 \%$ de los 68 jornales empleados fueron no remunerados (familiares), mientras que en San Lucas Tulcingo de 61 jornales, 53\% cubrieron esta condición. Respecto a la superficie, en la primera localidad cada productor tiene en promedio 3.3 hectáreas, mientras que en la segunda 2.1 hectáreas, superficie semejante a la reportada por Ayala (et al. 2014, 49) para 
Modo de apropiación de la naturaleza en las unidades de producción campesinas de amaranto y maíz en Tochimilco, Puebla, México

otras regiones amaranteras de Puebla, Morelos y Tlaxcala que siembra en promedio 2.52 ha, característica de la agricultura campesina.

Para ambas comunidades la agricultura es la actividad más importante en la generación de ingresos. En La Magdalena Yancuitlalpan 78.1\% de las UPC y en San Lucas Tulcingo 92.7\% dependen únicamente de la agricultura, aspecto que coincide con lo encontrado en otros estados por Ayala et al. $(2014,49)$.

\section{Diversidad de los subsistemas agrícolas y pecuarios}

En ambas comunidades los principales cultivos sembrados son maíz y amaranto, el primero tiene como destino primordial el autoconsumo, y el segundo se destina casi en su totalidad el mercado. En cuanto a la producción de maíz, considerando un consumo anual per cápita de $215 \mathrm{~kg}, 78.8 \%$ de las UPC en La Magdalena Yancuitlalpan y $92.7 \%$ en San Lucas Tulcingo tuvieron autosuficiencia alimentaria.

El amaranto es el cultivo comercial más importante para ambas localidades debido a los ingresos que genera para $93.3 \%$ de las UPC, y porque $35.2 \%$ lo emplea como ahorro. Se encontró que mientras su precio fluctúa a lo largo del año, la demanda por parte de los acopiadores se mantiene, por lo que los productores deciden cuánto y cuándo venderlo en su localidad. Otras cualidades de este producto es su precio, ya que este es más alto comparado con el maíz, y además se puede almacenar hasta por 5 años sin afectaciones de plagas y enfermedades postcosecha (Martínez 2016, 119). Durante el 2015 en la zona de estudio el amaranto se vendió en promedio a \$16,374.00 por tonelada. Para el mismo año FIRA $(2015,27)$ reportó para el maíz un precio promedio rural de $\$ 3,423.00$ por tonelada. Un estudio realizado para la misma zona, reporta que para el ciclo agrícola P-V 2013 el amaranto resultó con una Relación Beneficio/Costo (RB/C) de 3.52, mientras el maíz fue de 0.68 (Muñoz et al. 2017, 801).

Las UPC siembran diversos cultivos de autoconsumo y comerciales. En el primer grupo para el caso de La Magdalena Yancuitlalpan se cultivan huauzontle, haba, frijol, calabaza y cempasúchil, y entre los cultivos comerciales se producen aguacate y 11 tipos de flores; el máximo de especies manejadas por UPC fue de 20 y el promedio 11.2. En San Lucas Tulcingo los cultivos de autoconsumo fueron limón, calabaza, chile, frijol, haba, huaje y cempasúchil, y para venta tomate rojo y tomate verde; el máximo de especies manejadas fue 18 y el promedio 10.6 por UPC. Esta característica resalta la importancia de la diversidad y la complementariedad 
entre cultivos de autoconsumo y comerciales, la diversidad es una condición que se encuentra en otras unidades de producción amaranteras como es el caso de las pertenecientes a Tulyehualco, en el Distrito Federal (hoy Ciudad de México) (Méndez 2011, 78), al igual que en Morelos y otras regiones de Puebla, que además de amaranto se complementa con maíz, frijol, sorgo, cacahuate, hortalizas y trigo (Ayala et al. 2014, 49).

Respecto a la actividad pecuaria $97.3 \%$ de las UPC cuentan con animales. El promedio de especies manejadas es de tres para el caso de La Magdalena Yancuitlalpan y de 3.5 en San Lucas Tulcingo. El principal destino de la producción es el autoconsumo. Relacionando los aportes de la ganadería a la agricultura, se encontró que 63.5\% de las UPC aplicaron estiércol a sus parcelas; en el caso de La Magdalena Yancuitlalpan, 39.4\% aplicó más estiércol que fertilizantes químicos o abonos orgánicos adquiridos en el mercado. Al respecto, Sánchez et al. $(2016,99)$ señalan que el productor que realiza un manejo tradicional sabe que la ausencia de fertilizante químico no afecta si es compensado con abono de origen animal, y por el contrario, los productores que realizan prácticas del modo agroindustrial aplicaron mayor cantidad de fertilizante químico.

Estos resultados muestran que la diversificación de la UPC promueve su independencia respecto a insumos externos. Esta premisa también se refleja en la producción de sus propias semillas de amaranto y de maíz. Los productores identifican siete especies de amaranto (diversidad dentro de especies): amarilla, blanca, roja, pinta, payasita, moradita y verde, y cuatro especies diferentes de maíz (blanco, amarillo, morado y pozolero).

\section{Tecnología de producción}

Históricamente las sociedades tradicionales han generado sistemas cognitivos sobre su contexto, y han conservado un repertorio de conocimientos que se transmiten de generación en generación, generalmente es local, colectivo, diacrónico y holístico, ligado a sus necesidades prácticas para el uso y manejo de los ecosistemas locales. Este conocimiento tradicional sirve a los productores tradicionales para clarificar cómo perciben, conciben y conceptualizan los recursos y los paisajes o ecosistemas de los que dependen para subsistir, a través del diseño e implementación de estrategias de supervivencia (Toledo y Barrera 2008, 70-71). Sin embargo, los mecanismos modernizadores tienden a transformar el modo campesino (Toledo 1999, 12), como se muestra a continuación con las prácticas de cultivo del amaranto y del maíz: 
Las prácticas agrícolas son muy semejantes en las dos comunidades. La diferencia es el mayor uso de maquinaria en San Lucas Tulcingo, en relación a La Magdalena Yancuitlapan, por las condiciones topográficas de los terrenos. Para el cultivo de amaranto se realizan un barbecho con tractor y dos rastreos con yunta entre los meses de abril y mayo. Posteriormente se hace el surcado con yunta en la mayoría de los casos, la distancia entre surcos es de $80 \mathrm{~cm}$ y se realiza entre mayo y junio. La siembra se realiza en forma manual a finales de mayo y durante junio, pudiendo extenderse en ocasiones a principios de julio. La mayoría de los campesinos la realiza a mediado de junio. El procedimiento, dado el tamaño tan pequeño de la semilla, consiste en mezclar $8.0 \mathrm{~kg}$ de semilla criolla en promedio con 9 bultos de abono orgánico (estiércol de vacas o de aves) que alcanza para una hectárea, depositando un puño cada $25 \mathrm{~cm}$ aproximadamente en el lomo del surco. Una vez que nacen las plantas se realizan dos labores: la primera aproximadamente al mes de la siembra que se acompaña del raleo o deshije (selección de plantas), el deshierbe manual y la primera fertilización. En la segunda labor también se deshierba y se fertiliza. La fertilización en general consiste en aplicar 3 bultos (150 kg) de urea, 2 bultos (100 kg) de fosfato diamónico (18-46-00) y al menos 6 bultos de abono orgánico por hectárea en cada labor. Para controlar plagas y enfermedades aplican ocasionalmente productos químicos. La cosecha se realiza en forma manual entre noviembre y diciembre, cortando las dos terceras partes superiores de la planta, depositándola en el suelo y exponiéndola al sol para su secado. Finalmente se realiza la trilla con maquinaria (trilladora), entre 15 y 20 días después de la tumba. Se limpia el grano y se deposita en costales para su almacenamiento.

Para el caso del maíz existen dos sistemas de manejo: el marceño y el tardío. El marceño se siembra en la zona alta (más húmeda), por lo que en enero y febrero se prepara el suelo, realizando tres o más labores con yunta. Esta práctica permite captar humedad para sembrar antes de las lluvias (entre febrero y marzo) durante la luna llena. El surcado se realiza con yunta, con distancias entre surcos de $80 \mathrm{~cm}$, e inmediatamente se siembra, depositando entre dos y tres semillas cada $80 \mathrm{~cm}$. Una vez que nacen las plantas se realizan dos labores: la primera (aporque) aproximadamente al mes de la siembra que se acompaña del deshierbe y la primera fertilización. En la segunda labor (bajar tierra) solo se fertiliza (ya no requiere deshierbe). La fertilización en general consiste en aplicar 3 bultos $(150 \mathrm{~kg})$ de urea y 2 bultos $(100 \mathrm{~kg})$ de fosfato diamónico (18-46-00) por hectárea en cada labor. Para controlar plagas aplican insecticidas. La cosecha se 
realiza en forma manual entre enero y febrero con luna llena. Como se observa, el ciclo es largo, alrededor de un año.

El sistema tardío se realiza en las zonas más bajas del territorio. Se requiere de un barbecho con tractor y dos rastreos que se realizan con yunta durante los meses de abril y mayo. El surcado se realiza con yunta, con distancias entre surcos de $80 \mathrm{~cm}$ e inmediatamente se siembra, depositando entre dos y tres semillas cada 40-50 cm. Se siembra en abril y mayo en luna llena. Una vez que emergen las plantas se realizan dos labores: la primera (aporque) aproximadamente al mes de la siembra que se acompaña del deshierbe y la primera fertilización. En la segunda labor también se deshierba y se fertiliza. La fertilización en general consiste en aplicar 3 bultos $(150 \mathrm{~kg})$ de urea y 2 bultos $(100 \mathrm{~kg})$ de fosfato diamónico (18-46-00) por hectárea en cada labor. Para controlar plagas aplican insecticidas. La cosecha se realiza en forma manual entre enero y febrero cuando hay luna llena.

Las Unidades de Producción Campesinas (UPC) han desarrollado otros conocimientos para manejar su sistema de producción, como: a) la rotación de cultivos cada tercer ciclo (de no hacerlo será difícil el control de plagas), b) la aplicación de abonos orgánicos para mejorar la calidad de la tierra y obtener mejores rendimientos, c) la diversificación de cultivos, como estrategia de producción y disminución de riesgos, d) la siembra de diferentes cultivos en un mismo ciclo agrícola, e) la relación del proceso de producción con los movimientos lunares y, f) las relaciones entre el proceso productivo y las creencias divinas. En este conjunto de prácticas certifican su seguridad alimentaria.

Con este sistema de prácticas aparentemente sencillas y simples, los pequeños agricultores han creado o heredado sistemas agrícolas complejos de producción y consumo, que han sido la base para satisfacer sus necesidades, aún en condiciones adversas. Generalmente los insumos son de la región y el trabajo es realizado por el ser humano y por los animales (Altieri 1999, 103104). Los campesinos son los principales proveedores de alimentos para más del $70 \%$ de la población del mundo, y utilizan menos del 25\% de las tierras agrícola, menos del $20 \%$ del agua y 10\% de la energía fósil, además de cultivar 2.1 millones de variedades de plantas de las más de 7 mil especies de plantas domesticadas en el mundo (ETC Group 2017, 6, 17, 19). 
Modo de apropiación de la naturaleza en las unidades de producción campesinas de amaranto y maíz en Tochimilco, Puebla, México

\section{Fuente de energía empleada}

Con respecto al proceso productivo del amaranto, se aprecia en la Gráfica 1 que el uso de maquinaria agrícola fue mayor en San Lucas Tulcingo. En ambas localidades se realizaron en promedio 2.7 actividades para la preparación del suelo; sin embargo, en La Magdalena Yancuitlalpan un porcentaje mayor empleó yunta. En cuanto a los deshierbes, se realizaron en promedio 1.4, algunos se efectuaron junto con el raleo empleando yunta, otros en la primera y/o segunda labor. El 3\% en La Magdalena Yancuitlalpan y el 12\% en San Lucas Tulcingo emplearon herbicidas.

Gráfica 1. Tipo de energía empleada en el proceso productivo del amaranto en La Magdalena Yancuitlalpan y en San Lucas Tulcingo, Puebla.

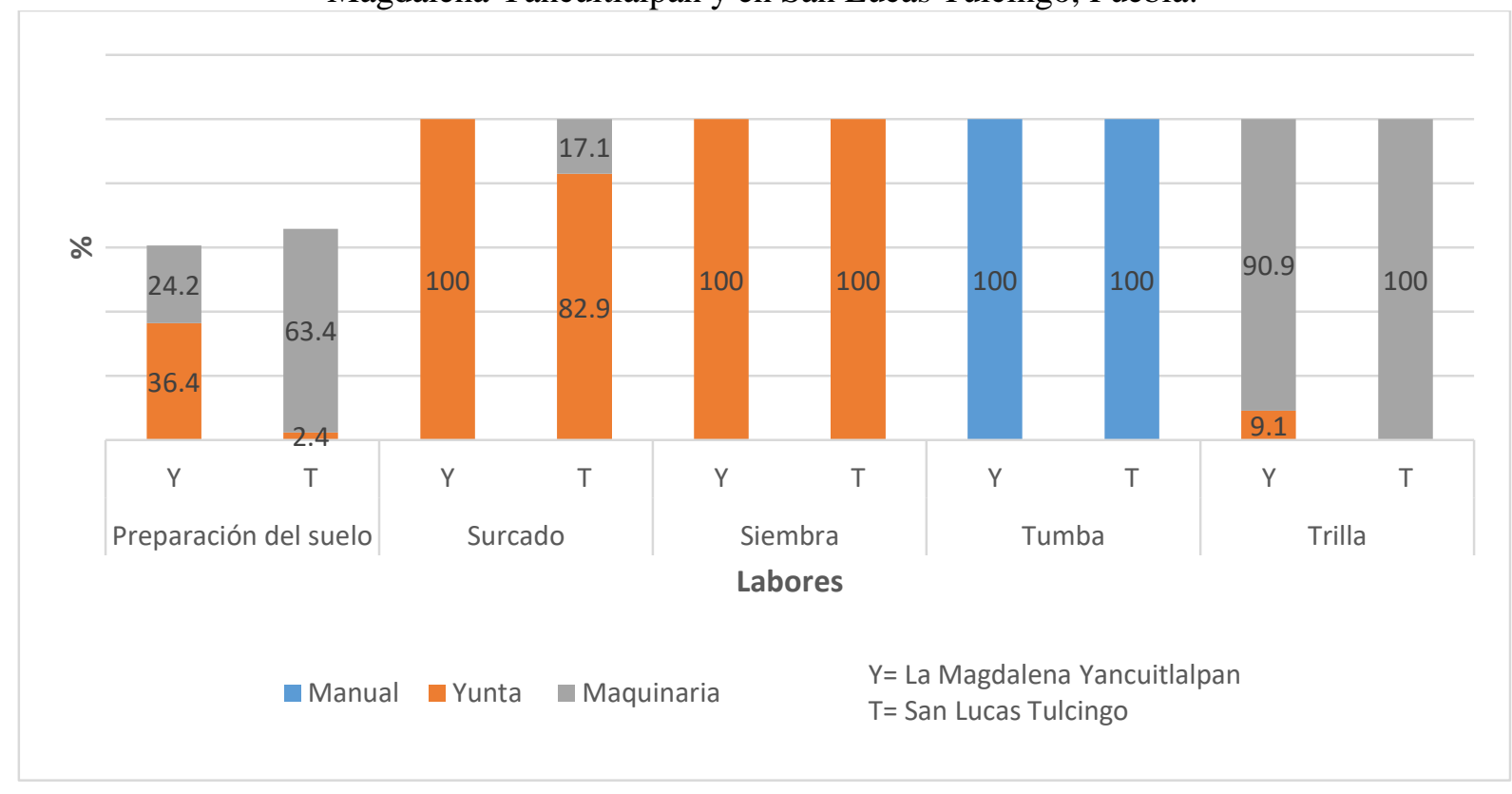

Fuente: Elaboración propia a partir de datos de campo.

\section{Índice de Campesinidad (IC)}

De acuerdo al Índice de Campesinidad calculado para ambas localidades se mantienen dentro de un rango campesino. Se encontró que existe diferencia estadística significativa $(\mathrm{t}=-2.031: \mathrm{p}=$ 0.047) entre las localidades estudiadas, resultando con un IC mayor en La Magdalena Yancuitlalpan (0.298) y menor en San Lucas Tulcingo (0.327) (Tabla 2), aún con esta diferencia, la tendencia es al modo de apropiación campesino. 
Tabla 2. Índice de campesinidad e indicadores en las Unidades de Producción Campesina del municipio de Tochimilco, Puebla, México.

\begin{tabular}{lcccccccccc}
\hline Localidad & FE & Esc & Dep & FT & Div & PT & PE & Con & Cos & IC \\
\hline $\begin{array}{l}\text { LMY } \\
\quad \begin{array}{l}\text { Medias } \\
\text { SLT }\end{array}\end{array}$ & 0.348 & 0.336 & 0.188 & 0.432 & 0.396 & 0.060 & 0.204 & 0.075 & 0.139 & 0.298 \\
$\quad 0.469$ & 0.216 & 0.195 & 0.354 & 0.402 & 0.080 & 0.194 & 0.048 & 0.239 & 0.327 \\
\hline $\mathrm{t}$ & -4.031 & 3.858 & -0.365 & 0.700 & -0.246 & -2.786 & -1.643 & 0.862 & -1.933 & -2.031 \\
$\mathrm{p}$ & 0.000 & 0.000 & 0.716 & 0.486 & 0.806 & 0.007 & 0.105 & 0.392 & 0.057 & 0.047 \\
\hline
\end{tabular}

Nota: LMY= La Magdalena Yancuitlalpan; SLT $=$ San Lucas Tulcingo; FE= Fuente de Energía; Esc $=$ Escala; Dep $=$ Dependencia; $F T=$ Fuerza de Trabajo; Div = Diversidad; $P T=$ Productividad del Trabajo; $\mathrm{PE}=$ Productividad Energética; Con= Conocimiento; Cos= Cosmovisión; IC = Índice de Campesinidad.

Fuente: Elaboración propia a partir de los datos de campo.

El indicador Fuente de Energía (FE) muestra diferencia estadística, observándose un valor mayor en San Lucas Tulcingo, lo que podría calificarse con tendencia al modo agroindustrial; este indicador tiene particular importancia ya que de él se derivan otros como Productividad del Trabajo (PT) que expresa la relación entre la mano de obra requerida (en menor cantidad ante la adopción de tecnología) y el rendimiento obtenido por hectárea; así como la Productividad Energética (PE) que refleja la relación entre la energía invertida (que es mayor mediante el uso de maquinaría) y el rendimiento obtenido por hectárea.

Para reconocer las características de mayor relación con el Índice de Campesinidad (IC), se aplicó un modelo de regresión logística. Utilizando un procedimiento de selección de variables con el método de Wald hacia adelante, resultando que la Fuerza de Trabajo (FT) y la Fuente de Energía (FE) fueron los indicadores que resultaron significativos (Tabla 3), lo que manifiesta su asociación con el IC.

Por lo tanto, la fuerza de trabajo familiar es determinante para mantener el modo campesino, mientras el incremento del empleo de energía fósil propiciada por el uso de maquinaria agrícola fomenta la tendencia al modo de apropiación agroindustrial, aunque de acuerdo con Cáceres et al. $(2010,115)$ la intensificación dada en este caso por mayor empleo de maquinaria agrícola puede representar una estrategia defensiva más que una estrategia de adopción del modo agroindustrial, que intenta atenuar los impactos generados por el modelo de desarrollo dominante. 
Modo de apropiación de la naturaleza en las unidades de producción campesinas de amaranto y maíz en Tochimilco, Puebla, México

Tabla 3. Estimadores del modelo de regresión logística con el método de selección por pasos adelante (Wald).

\begin{tabular}{lcccc}
\hline \multicolumn{1}{c}{ Variables } & Estimador & $\begin{array}{c}\text { Error } \\
\text { Estándar }\end{array}$ & Wald & P \\
\hline Fuerza de Trabajo & 27.99 & 13.79 & 4.12 & 0.042 \\
Fuente de Energía & 8.18 & 2.90 & 7.94 & 0.005 \\
\hline Constante & -20.07 & 7.75 & 6.71 & 0.010 \\
\hline
\end{tabular}

Fuente: Elaboración propia estimado con datos de campo.

El indicador Fuerza de Trabajo (FT) expresa la proporción de jornales remunerados y no remunerados en el cultivo de amaranto y el número de labores donde se emplea mano de obra remunerada en maíz; en promedio en La Magdalena Yancuitlalpan considerada como campesino (de 0 a 0.49 ) de acuerdo al IC, $52.4 \%$ de la mano de obra empleada en amaranto es familiar y en San Lucas Tulcingo 37.2\%, mientras el número de labores donde se emplea mano de obra remunerada en maíz en ambas localidades en promedio fueron de 1.3.

En este sentido, Wanderley $(2013,48)$ menciona que la base de la agricultura campesina está dada por las unidades de producción administradas por la familia y que aun integrándose al mercado con productos comerciales como en este caso el amaranto, el agricultor sigue un manejo campesino en la medida que la familia define las estrategias de producción y reproducción.

Por otra parte, el indicador Fuente de Energía (FE) se compone de: a) la proporción del empleo de fuerza humana, yunta y maquinaria utilizada para realizar las labores en el amaranto, b) del empleo de maquinaria empleada en el cultivo de maíz, y c) de la fuente de energía para cocinar en las UPC. Se encontró diferencia en el promedio de labores realizadas con tractor; en en La Magdalena Yancuitlalpan con más tendencia a la campesinidad se realizan 2.7, mientras que en San Lucas Tulcingo con menor campesinidad se realizan 3.8 labores. El amaranto es un cultivo comercial, la introducción de tecnología y la búsqueda del aumento de la productividad representan un intento por competir con los agroecosistemas industriales (Figueroa 2005, 39).

Los sistemas agrícolas que utilizan maquinaria son más demandantes de energía fósil y la eficiencia es menor respecto a la biomasa seca cosechada, como en el caso del cultivo de maíz analizado por Sánchez et al. $(2014,116)$. Estos sistemas tienden a un mayor desorden o entropía, y para crear orden es necesario gastar energía (Flores y Sarandón 2014, 193), por lo que los agroecosistemas basados en aportes de energía del trabajo humano o animal son más eficientes 
energéticamente (Gliessman 2001, citado por Flores y Sarandón 2014, 202); sin embargo, la energía consumida depende del cultivo y la tecnología utilizada (Flores y Sarandón 2014, 204).

Otro aspecto que fortalece al modo de apropiación campesino es la diversidad biológica de especies, tanto agrícolas como pecuarias. Las sociedades campesinas históricamente han desarrollado sistemas de manejo de los recursos, donde la diversidad es a la vez una condición para garantizar el suministro continuo de energía y materiales, resultado de su adaptación a condiciones precisas de cada lugar y momento dentro del agroecosistema (Acosta 2008, 2). Esta diversidad a su vez se asocia con los beneficios observados en la zona de estudio que coincide con lo señalado por Altieri (1999, 107-108): baja población de plagas y enfermedades, cubierta del suelo, aumento en las oportunidades de comercialización y abastecimiento seguro de una gama importante de productos e incorporación de estiércol del corral a las parcelas de cultivo. Para los campesinos la diversidad funciona como un seguro para enfrentar el cambio ambiental y las necesidades sociales y económicas, por lo que su estrategia es sembrar varios especies y variedades de cultivos para estabilizar los rendimientos y diversificar su dieta (Nicholls y Altieri 2012, 32).

Por otra parte en los paisajes relativamente homogéneos predomina la producción agroindustrial donde las UPC pasan a depender de fuentes de energía fósiles sustituyendo a la animal y humana (Acosta 2008, 6; Gliessman 2002, 230) o como mencionan Casado et al. (2000, 37) que el modo agroindustrial tiene lugar como consecuencia de la sustitución paulatina de la reposición interna de energía por la adquisición de energía exterior. Tal es el caso de San Lucas Tulcingo donde el paisaje es aparentemente más homogéneo por sus pendientes poco pronunciadas que permite el uso de maquinaria agrícola en un mayor número de labores, en comparación con La Magdalena Yancuitlalpan con pendientes medianamente pronunciadas donde resulta más difícil el uso de maquinaria y se conserva el trabajo humano y con tracción animal. Sin embargo, otros factores que originan el bajo índice de mecanización para la producción de amaranto y de maíz en los estados de Puebla y Morelos, puede atribuirse al tamaño de la superficie (menor a las $5 \mathrm{ha}$ ), a la maquinaria inadecuada para estas superficie y al alto costo de la misma (Ayala et al. 2016, 78).

El Índice de Campesinidad de 0.298 para La Magdalena Yancuitlalpan y de 0.327 para San Lucas Tulcingo, muestran la tendencia al modo de apropiación campesino; esto significa que las unidades de producción campesinas de ambas comunidades están arraigadas a la actividad 
Modo de apropiación de la naturaleza en las unidades de producción campesinas de amaranto y maíz en Tochimilco, Puebla, México

agrícola con dependencia de mano de obra familiar para producir, tanto para el autoconsumo, como para el mercado. Utilizan como estrategia la diversidad de cultivos y especies pecuarias, y su cultura productiva está relacionada con creencias del universo y místicas. Sin embargo, San Lucas Tulcingo ha incorporado y ensamblado al proceso de producción conocimientos e insumos exógenos.

\section{Conclusiones}

El modo de apropiación campesino sigue siendo pertinente ecológica, social y económicamente en ambas comunidades. El Índice de Campesinada (IC) resultó mayor en La Magdalena Yancuitlalpan (0.298) que en San Lucas Tulcingo (0.327). Considerando los nueve atributos del Índice de Campesinidad (IC), para la primera comunidad todos fortalecieron la continuidad del modo de apropiación campesino: fuente de energía (doméstica y productiva), la escala (tamaño de la UPC), dependencia (alimentaria y productiva), fuerza de trabajo (tipo), diversidad (agrícola y pecuaria), productividad del trabajo, productividad energética (eficiencia energética), conocimientos (tipo) y cosmovisión (creencias); para la segunda comunidad, la fuente de energía fue el único indicador que modificó el modo de apropiación campesino, el resto lo fortalecieron.

La característica que marca la diferencia en el modo campesino destaca la fuerza de trabajo predominantemente familiar, lo cual favoreció a este modo de apropiación, mientras que en el modo agroindustrial, la fuente de energía con una tendencia importante al empleo de energía fósil por el uso de maquinaria agrícola en las labores de cultivo diferenció el modo campesino.

Esto muestra que las comunidades San Lucas Tulcingo y La Magdalena Yancuitlalpan del municipio de Tochimilco, Puebla, México, presentaron rasgos del modo de manejo campesino, no obstante que la primera mostró una mínima tendencia al modo de apropiación agroindustrial al usar como principal fuente de energía la derivada del petróleo, debido al uso de maquinaria en actividades agrícolas. Esta práctica está relacionada con la morfología del paisaje. En San Lucas Tulcingo los terrenos agrícolas fueron más homogéneos en relación a La Magdalena Yancuitlalpan que presentó pendientes escarpadas.

La morfología del paisaje es un elemento que debe considerarse en la transformación de las unidades de producción, sumado al tipo de cultivo, ya sea para autoconsumo o para el mercado. También es importante considerar que existe cierto grado de transformación de los 
ecosistemas hacia el modo agroindustrial. Las prácticas que se introdujeron de la agricultura industrial son ajustes para conservar las estrategias de producción y de reproducción de la unidad de producción campesina ante un contexto cambiante.

Se recomienda continuar con estudios que permitan comprender la adaptación de las UPC ante las diferentes presiones que ejerce el ambiente al que deben ajustarse, sin perder su esencia y razón de ser en el que han construido su estilo de vida. Esto permitirá plantear estrategias alternativas para el fortalecimiento del modo de apropiación campesino y la conservación de los recursos base de los que dependen.

\section{Agradecimientos}

Se agradece al Consejo Nacional de Ciencia y Tecnología (CONACYT) por la beca otorgada, al Colegio de Postgraduados por el apoyo académico y a los productores de Tochimilco, Puebla, por compartir su información para esta investigación.

\section{$\underline{\text { Referencias }}$}

Acosta Naranjo, Rufino. (2008). La biodiversidad en la agricultura: la importancia de las variedades locales. En Nuevas rutas para el desarrollo en América Latina. Experiencias globales y locales, compilado por Juan Maestre, Alba González y Ángel Casas, $1^{\text {a }}$ ed., 239260. México: Universidad Iberoamericana.

Altieri, Miguel. (1999). Agroecología: bases científicas para una agricultura sustentable. Montevideo: Nordan-Comunidad.

Altieri, Miguel y Nicholls, Clara. (2000). Agroecología: teoría y práctica para una agricultura sustentable. $1^{\text {a }}$ edición. PNUMA.

Ayala Garay, Alma Velia; Rivas Valencia, Patricia; Cortes Espinoza, Lorena; De la O Olán, Micaela; Escobedo López, Diana y Espitia Rangel, Eduardo. (2014). La rentabilidad del cultivo de amaranto (Amaranthus spp.) en la región centro de México. Ciencia Ergo-Sum, Ciencias Naturales y Agropecuarias 21(1): 47-54.

Ayala Garay, Alma Velia; González González, Miguel y Limón Ortega, Agustín. (2016). Mecanización del proceso de producción de maíz y amaranto en la región centro de México. Revista Ciencias Técnicas Agropecuarias, 25(4): 74-80. 
Modo de apropiación de la naturaleza en las unidades de producción campesinas de amaranto y maíz en Tochimilco, Puebla, México

Cáceres, Daniel M.; Soto, Gustavo; Ferrer, Guillermo; Silvetti, Felicitas y Bisio, Catalina. (2010). La expansión de la agricultura industrial en Argentina Central. Su impacto en las estrategias campesinas. Cuadernos de desarrollo rural, Bogotá, 7(64): 91-119.

Camejo Pereira, Viviane. (2016). Agrobiodiversidade ameacada: os direitos dos agricultores e os riscos da contaminacao transgenica. En Desenvolvimento, agricultura e sustentabilidade, compolado por Fábio Dal Soglio y Rumi Kubo. $1^{\text {a }}$ edición, 75-92. Brasil: UFRGS.

Carrasco Aquino, Roque. (2006). La naturaleza y sus formas de apropiación en contradicción. Mundo Siglo XXI 6: 55-65.

Casado Guzmán, Gloria; González de Molina, Manuel y Sevilla Guzmán, Eduardo. (2000). Introducción a la agroecología como desarrollo rural sostenible. Madrid: Mundi-Prensa.

Chayanov, Alexander V. (1985). La organización de la unidad económica campesina. Ediciones Nueva Visión, Buenos Aires.

CONEVAL. (2010). Informe anual sobre la situación de pobreza y rezago social. Tochimilco, Puebla. SEDESOL.

Dal Soglio, Fábio. (2016). A agricultura moderna e o mito da produtividade. En Desenvolvimento, agricultura e sustentabilidade. Compilado por Fábio Dal Soglio y Rumi Kubo, $1^{\text {a }}$ edición, 11-38. Brasil: UFRGS.

ETC Group. (2017). ¿Quién nos alimentará? ¿La red campesina alimentaria o la cadena agroindustrial? $3^{\circ}$ Edición, 2017.

http://www.etcgroup.org/sites/www.etcgroup.org/files/files/etc-quiennosalimentara-2017es.pdf (7 de marzo, 2018).

European Coordination Vía Campesina. (2018). ¡Soberanía alimentaria ya!. Una guía por la soberanía alimentaria.

https://viacampesina.org/en/wp-content/uploads/sites/2/2018/02/Food-Sovereignty-a-guideES-version-low-res.pdf (2 de marzo, 2018).

FAO. (2015). El estado de la inseguridad alimentaria en el mundo 2015. Cumplimiento de los objetivos internacionales para 2015 en relación con el hambre: balance de los desiguales progresos. Roma: FAO.

Flores, Claudia C. y Sarandón, Santiago J. (2014). La energía en los agroecosistemas. En Agroecología: bases teóricas para el diseño y manejo de agroecosistemas sustentables. 
Sarandón, Santiago J. y Flores, Claudia C. (Editores), $1^{\text {a }}$ ed. La Plata: Universidad Nacional de La Plata. Buenos Aires Argentina.

Figueroa, Víctor Manuel. (2005). América Latina: descomposición y persistencia de lo campesino. Problemas del desarrollo 36(142): 27-50.

FIRA. (2015). Panorama agroalimentario. Maíz 2016. Dirección de investigación y evaluación económica y sectorial.

https://www.gob.mx/cms/uploads/attachment/file/200637/Panorama_Agroalimentario_Ma_ Z_2016.pdf (6 de noviembre, 2017).

Gabriel, Dooren; Roschewitz, Indra; Tscharntke, Teja and Thies, Cartens. (2006). Beta diversity at different spatial scales: Plant communities in organic and conventional agriculture. Ecological Applications 16(5): 2011-2021.

Gallopín, Gilberto; Thrupp, Lori Ann; Kaimowitz, David; da Veiga, José Elí; Trigo, Eduardo; Altieri, Miguel; Bárcena, Alicia y Toledo, Víctor M. (1995). Semillas para el futuro: agricultura sostenible y recursos naturales en las Américas. Costa Rica: IICA.

Gliessman, Stephen. (2002). Agroecología: procesos ecológicos en agricultura sostenible. Costa Rica: CATIE.

Hernández, Eva. (2013) La conservación de la biodiversidad en los sistemas agrarios. Ecosistemas 22(1):1-4.

Hernández Sampieri, Roberto; Fernández Collado, Carlos y Baptista Pilar, Lucio. (2006). Metodología de la investigación, 4ª ed. México: McGraw-Hill.

INAFED. (2010). Enciclopedia de los municipios y delegaciones de México. Estado de Puebla. http://www.inafed.gob.mx/work/enciclopedia/EMM21puebla/municipios/21188a.html (7 de noviembre de 2017).

INEGI. (2010). Compendio de información geográfica municipal 2010 de los Estados Unidos Mexicanos, Tochimilco, Puebla. Clave geoestadística 21188.

http://www.inegi.org.mx/geo/contenidos/topografia/compendio.aspx (28 de octubre de 2017).

INEGI. (2017). Marco geoestadístico-Datos vectoriales.

Martínez Castillo, Róger. (2002). Agroecología: atributos de sustentabilidad. Inter Sedes, III(5): $25-45$. 
Modo de apropiación de la naturaleza en las unidades de producción campesinas de amaranto y maíz en Tochimilco, Puebla, México

Martínez Salvador, Laura. (2016). Seguridad alimentaria, autosuficiencia y disponibilidad del amaranto en México. Problemas del desarrollo 186(47): 107-132.

Menchú, María Teresa y Méndez, Humberto. (2007). Tabla de composición de alimentos de Centroamérica. $2^{\mathrm{a}}$ edición. Guatemala: INCAP.

Méndez Bautista, Clara Luz. (2011). Caracterización de la unidad agroindustrial familiar amarantera de Santiago Tulyahualco, D.F. México. Colegio de Postgraduados.

Muñoz Máximo, Tania; Ocampo Fletes, Ignacio; Parra Inzunza, Filemón; Cervantes Vargas, Juana; Argumedo Macías, Adrián y Cruz Ramírez, Susana. (2017). Proceso de producción y mecanismos de comercialización de chía (Salvia hispánica L.) por familias campesinas de los municipios de Atzitzihuacán y Tochimilco, Puebla, México. Nova Scientia, No. 19, Vol. 9(2): 788-818.

Nicholls, Clara Inés y Altieri, Miguel A. (2012). Modelos ecológicos y resilientes de producción agrícola para el siglo XXI. Agroecología 6: 29-37.

Ortega Cerdâ, Miquel y Rivera Ferre, Marta G. (2010). Indicadores internacionales de soberanía alimentaria. Nuevas Herramientas para una nueva agricultura. Iberoamericana de Economía Ecológica 14: 53-77.

Rappaport, Roy. (1975). El flujo de energía en una sociedad agrícola. En Biología y cultura: introducción a la antropología biológica y social, compilado por Jorgensen G. Joseph 378391. Madrid: H. Blume.

Rojas Soriano, Raúl. (2013). Guía para realizar investigaciones sociales. Trigésima octava edición. México: Plaza y Valdés.

SAGARPA. (2016). Listado de beneficiarios PROCAMPO.

http://www.sagarpa.gob.mx/agricultura/Programas/proagro/procampo/Beneficiarios/Pagina s/2013.aspx (marzo de 2016).

SAGARPA y FAO. (2012). Agricultura familiar con potencial productivo en México.

Sánchez Olarte, Josset; Argumedo Macías, Adrián; Álvarez Gaxiola, J. Felipe; Méndez Espinoza, J. Arturo y Ortiz Espejel, Benjamín. (2016). Análisis económico del sistema sociotécnico del cultivo de amaranto en Tochimilco, Puebla. Acta Universitaria 26(3): 95-104.

Sánchez Morales, Primo; Ocampo Fletes, Ignacio; Parra Inzunza, Filemón; Sánchez Escudero, Julio; María Ramírez, Andrés y Argumedo Macías, Adrián. (2014). Evaluación de la 
sustentabilidad del agroecosistema maíz en la región Huamantla, Tlaxcala, México. Agroecología 9 (1 y 2): 111-122.

Sarandón, Santiago. (2011). La agroecología: su rol en el logro de una agricultura sustentable. Argentina: Universidad de la Plata.

Sevilla Guzmán, Eduardo y Soler Motiel, Marta. (2009). Del desarrollo rural a la agroecología: hacia un cambio de paradigma. Documentación Social, 155: 23-39.

Sevilla, Eduardo y Woodgate, Graham. (2013). Agroecología: fundamentos del pensamiento social agrario y teoría sociológica. Agroecología, 8(2): 27-34.

Shanin, Teodor. (1983). La clase incómoda. España: Alianza Editorial, S. A.

Suso, M. J.; Bocci, R. y Chable, V. (2013). La diversidad, una herramienta poderosa para el desarrollo de una agricultura de bajos-insumos. Ecosistemas, 22(1):10-15.

SIAP. (2014). Agricultura, producción anual. http://www.siap.gob.mx/agricultura-produccionanual/ (octubre de 2015).

Toledo, Víctor. (1992). La racionalidad ecológica de la producción campesina. Agroecología y desarrollo, 5(6). http://www.clades.cl/revistas/5/rev5art3.htm (enero de 2016).

Toledo, Víctor. (1999). Campesinidad, agroindustrialidad, sostenibilidad: los fundamentos ecológicos e históricos del desarrollo rural. Revista de geografía agrícola.

Toledo, Víctor; Alarcón Cháires, Pablo y Barón, Lourdes. (2002a). La modernización rural en México: un análisis socioecológico. $1^{\mathrm{a}}$ ed. México: INE-SEMARNAT-UNAM.

Toledo, Víctor; Alarcón Cháires, Pablo y Barón, Lourdes. (2002b). Revizualizar lo rural: un enfoque socioecológico. Gaceta Ecológica, núm. 62: 7-20.

Toledo, Víctor y Barrera-Bassols, Narciso. (2008). La memoria biocultural. La importancia ecológica de las sabidurías tradicionales, $1^{\text {a }}$ ed. España: Icaria editorial.

Vara, Isabel y Cuellar, Mamen. (2013). Biodiversidad cultivada: una cuestión de coevolución y transdisciplinariedad. Ecosistemas 22(1): 5-9.

Wanderley, María. (2013). Agricultura familiar e campesinato: rupturas e continuidade. Estudos Sociedade e Agricultura, 1: 42-61.

Wolf, Eric R. (1982). Los campesinos. $3^{\text {a }}$ ed. $1^{\text {a }}$ reimpresión. Editorial Labor S. A. Barcelona. 